\title{
Age-period-cohort Analysis of Cardiovascular Disease Mortality in Japan, 1995-2018
}

\author{
Tasuku Okui \\ Medical Information Center, Kyusyu University Hospital, Fukuoka, Japan
}

Objectives: This study aimed to analyze the mortality of heart disease (HD), ischemic heart disease (IHD), and cerebrovascular disease (CeVD) through an age-period-cohort (APC) analysis.

Methods: We used data on mortality due to cardiovascular disease from 1995 to 2018 in Japan, as determined by Vital Statistics. Age groups from 0 years to 99 years were defined by 5 -year increments, and cohorts were defined for each age group of each year with a 1-year shift. We used Bayesian APC analysis to decompose the changes in the diseases' mortality rates into age, period, and cohort effects.

Results: The period effects for all diseases decreased during the analyzed periods for both men and women. The cohort effects for men increased substantially in cohorts born from around 1940 to the 1970s for all types of cardiovascular diseases. The cohort effects of HD decreased in the cohorts born in the 1970s or later for both men and women. Regarding IHD and CeVD, either a non-increase or decrease of cohort effects was confirmed for cohorts born in the 1970s or later for men, but the effects for women showed a continuously increasing trend in the cohorts born in the 1960s or later.

Conclusions: The cohort effects for IHD and CeVD showed increasing trends in younger generations of women. This suggests that preventive approaches against cardiovascular diseases are needed, particularly for women.

Key words: Cohort effect, Mortality, Japan, Cardiovascular diseases

\section{INTRODUCTION}

Cardiovascular diseases (CVDs), including heart disease (HD) and cerebrovascular disease (CeVD), are among the leading causes of death in Japan [1]. Among the various forms of HD, ischemic heart disease (IHD) is closely related to an individual's lifestyle, as is CeVD, and trends in IHD by itself are usually a fo-

Received: February 10, 2020 Accepted: March 26, 2020

Corresponding author: Tasuku Okui, PhD

Medical Information Center, Kyusyu University Hospital, Maidashi

3-1-1 Higashi-ku, Fukuoka 812-8582, Japan

E-mail: task10300@gmail.com

This is an Open Access article distributed under the terms of the Creative Commons Attribution Non-Commercial License (https://creativecommons.org/licenses/by$\mathrm{nc} / 4.0 / /$ which permits unrestricted non-commercial use, distribution, and reproduction in any medium, provided the original work is properly cited. cus of attention [2-5]. The numbers of IHD and CeVD patients are decreasing throughout Japan [6]. The estimated number of IHD patients decreased from 1.19 million in 1996 to 0.72 million in 2017, and that of CeVD patients decreased from 1.73 million in 1996 to 1.12 million in 2017. In addition, it has been reported that the age-adjusted mortality rates for HD and coronary heart disease are decreasing for those born after 1970 $[7,8]$. The age-adjusted mortality per 100000 persons for men decreased from around 160 in 1970 to around 60 in 2015. A decrease was seen for women from around 120 in 1970 to around 40 in 2015. However, the birth cohort effects for the current young generations remain unknown.

Age-period-cohort (APC) analyses are often used to investigate disease trends [9]. This method decomposes changes in statistics into age, period, and cohort effects. Although an APC 
analysis for HD has not been conducted in Japan, APC analyses have already been conducted for CeVD and IHD in Japan $[2,10]$ and other countries $[3-5,11,12]$. However, the data used in those studies were from 1955 to 2000 [2] and from 1960 to 2000 , respectively [10]. The cohorts that were analyzed were born between 1871 and 1970, whereas the cohort effects in younger generations have not been investigated yet.

Further, although previous studies used stratified APC models $[2,10]$, the estimated age effects did not reflect the increase in mortality rate with age. Therefore, some assumptions of those models may not be consistent with the data. In a study investigating trends in CVD mortality using data from 1950 to 2010 [13], all CVDs were analyzed altogether, meaning that potential differences according to the type of CVD were not taken into account. Furthermore, that study used a sequential method when estimating the APC model parameters, and the cohort effects were not determined uniquely. Although results from 2 models were shown for the cohort effects in the previous study [13], which method produced a more accurate estimate could not be fully elucidated.

We used a Bayesian APC model, as it takes into account the continuity of effects among adjacent time points. By using the Bayesian APC analysis method, we can estimate each parameter uniquely, without the problem of parameter identifiability, which often arises when using frequentist methods. In this study, we conducted an APC analysis of the mortality rates of $H D$, IHD, and CeVD in Japan. Although IHD is a form of HD, we conducted a separate analysis of IHD because IHD trends can differ from those of HD.

\section{METHODS}

We used data from Vital Statistics in Japan [14], which tallies the numbers of births, deaths, marriages, and other demographic factors in Japan. In Japan, death certificates are completed by physicians and submitted to municipalities, as is required by law. Death reports are collected in each municipality and in health centers throughout Japan. All of the information from death reports is finally sent to the Ministry of Health, Labour and Welfare of Japan [15].

We analyzed the yearly HD, IHD, and CeVD mortality and the total population number by age group and gender from 1995 to 2018. The International Classification of Diseases, 10th revision (ICD-10) codes were put into practice in 1995. Additionally, death certificates were also changed in 1995 [16]. The mor- tality statistics for CVDs also changed after this period. Therefore, we used data from 1995 in this study. We used the following ICD-10 codes to classify deaths caused by CVD: 101-I02.0, 105-I09, I20-125, I27, and I30-I52 for HD; I20-I25 for IHD; and 160-169 for CeVD. Hypertensive diseases were not included as HD.

First, we calculated the yearly mortality of HD, IHD, and CeVD per 100000 people, as well as the age-adjusted yearly mortality of HD, IHD, and CeVD per 100000 people. The ageadjusted yearly mortality of the diseases was calculated using the direct method [17]; the men population in 1995 was used as the standard population.

We used a Bayesian APC model [9] for the analysis. A firstorder correlated random-walk was used to identify parameters as the Bayesian prior distributions for each effect. Although a second-order correlated random-walk is occasionally used with Bayesian APC models, in our study, the parameters could not be uniquely identified. Therefore, we used the Hamiltonian Monte Carlo method to estimate the parameters. All statistical analyses were conducted using R version 3.5.1 (https:// cran.r-project.org/bin/windows/base/old/3.5.1/). Programs for the Bayesian APC model were written by the author using rstan software (https://rdrr.io/cran/rstan/), and figures were created by using ggplot2 (https://ggplot2.tidyverse.org/) and cowplot (https://cran.r-project.org/web/packages/cowplot/index.html). In order to evaluate the fit of the model, we compared the APC model with the following models: an age model (an APC model that does not contain period or cohort effects), an age-period model (an APC model that does not contain cohort effects), and an age-cohort model (an APC model that does not contain period effects).

The age groups were defined from 0-4 years to 95-99 years by 5 -year increments in the data. However, population data for the age group of 90-99 years could not be obtained for some years. Therefore, those who were 90-94 years old in 1995 (i.e., those who were born in 1901-1905) were the first cohort, as this was the oldest birth cohort in the data set. Through a 1-year shift of the birth years, starting from the first cohort, the age group of 0-4 years in 2018 (i.e., those who were born in 2014-2018) was the last cohort.

\section{Ethics Statement}

The institutional review board approval is not required because we analyzed the data that are available to the public. 

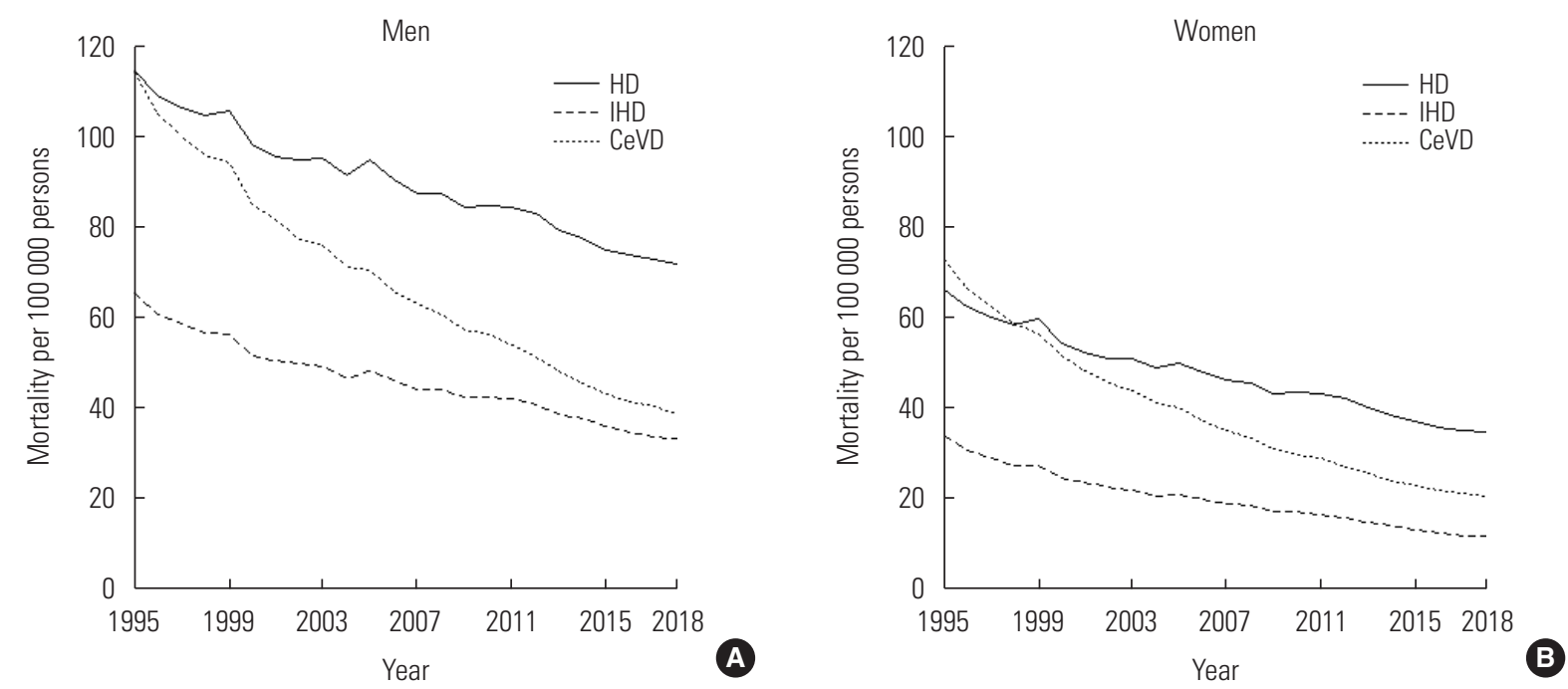

Figure 1. The age-adjusted yearly mortality rates of men (A) and women (B) for heart disease (HD), ischemic heart disease (IHD), and cerebrovascular disease (CeVD) per 100000 persons from 1995 to 2018.

\section{RESULTS}

Supplemental Material 1 shows the yearly mortality of $\mathrm{HD}$, IHD, and CeVD per 100000 people. The mortality due to HD increased throughout the analyzed period, and the degree of increase was larger for women than for men. Although the mortality due to IHD showed a slightly increasing trend for men, mortality for women showed a slightly decreasing trend. Mortality due to CeVD decreased throughout the analyzed periods for both men and women.

Figure 1 shows the age-adjusted yearly mortality rates of HD, IHD, and CeVD per 100000 people. The mortality rates of all diseases decreased throughout the analyzed period, and all mortality rates were consistently lower for women than for men. The most substantial decrease was seen in the mortality rates for CeVD in both men and women.

Supplemental Material 2 shows the model fit statistics for all the models. The Deviance information criterion (DIC) is a criterion for evaluating Bayesian models. Lower values of the DIC indicate better model fit. For most of the combinations of disease and gender, the DIC of the APC model was the smallest.

Figure 2 shows the results of the APC analysis for mortality in men due to HD, IHD, and CeVD. The change in the age effect was more substantial than the changes in other effects, and the effects exponentially increased from around the age of 50 years. The period effect decreased from 1995 to 2018 for all types of diseases. The cohort effect on HD showed an increasing trend from the earliest cohort until the cohorts born around 1970. In contrast, focusing on IHD, the cohort effect showed a decreasing trend from the earliest cohort until the cohorts born around 1940, which then increased until the cohorts born around 1970. Then, the cohort effect decreased until the cohort born in 1995 and remained flat after that. The cohort effect for CeVD also decreased from the earliest cohort until the cohorts born around 1940 and then increased until the cohorts born around 1980. Thereafter, the effect showed a gradually decreasing trend.

Figure 3 shows the results of the APC analysis for mortality in women due to HD, IHD, and CeVD. The trends of the age and period effects were similar to those of men. However, the rate ratio of the age effect became higher in older ages for women than for men. The cohort effect on HD fluctuated until the cohorts born around 1970, after which a decreasing trend was observed. The effect on IHD showed a decreasing trend from the start until around the cohort born in 1948, stabilized for some time, and then showed a gradually increasing trend starting around the cohort born in 1960. The cohort effect on CeVD showed relatively similar trends to those of IHD.

\section{DISCUSSION}

In this study, we conducted APC analyses of the mortality rates of HD, IHD, and CeVD. Our findings highlight changes in cohort effects from those who were born in the early 20th century to those born in the early 21 st century.

The results of the APC analysis showed that the scale of the 

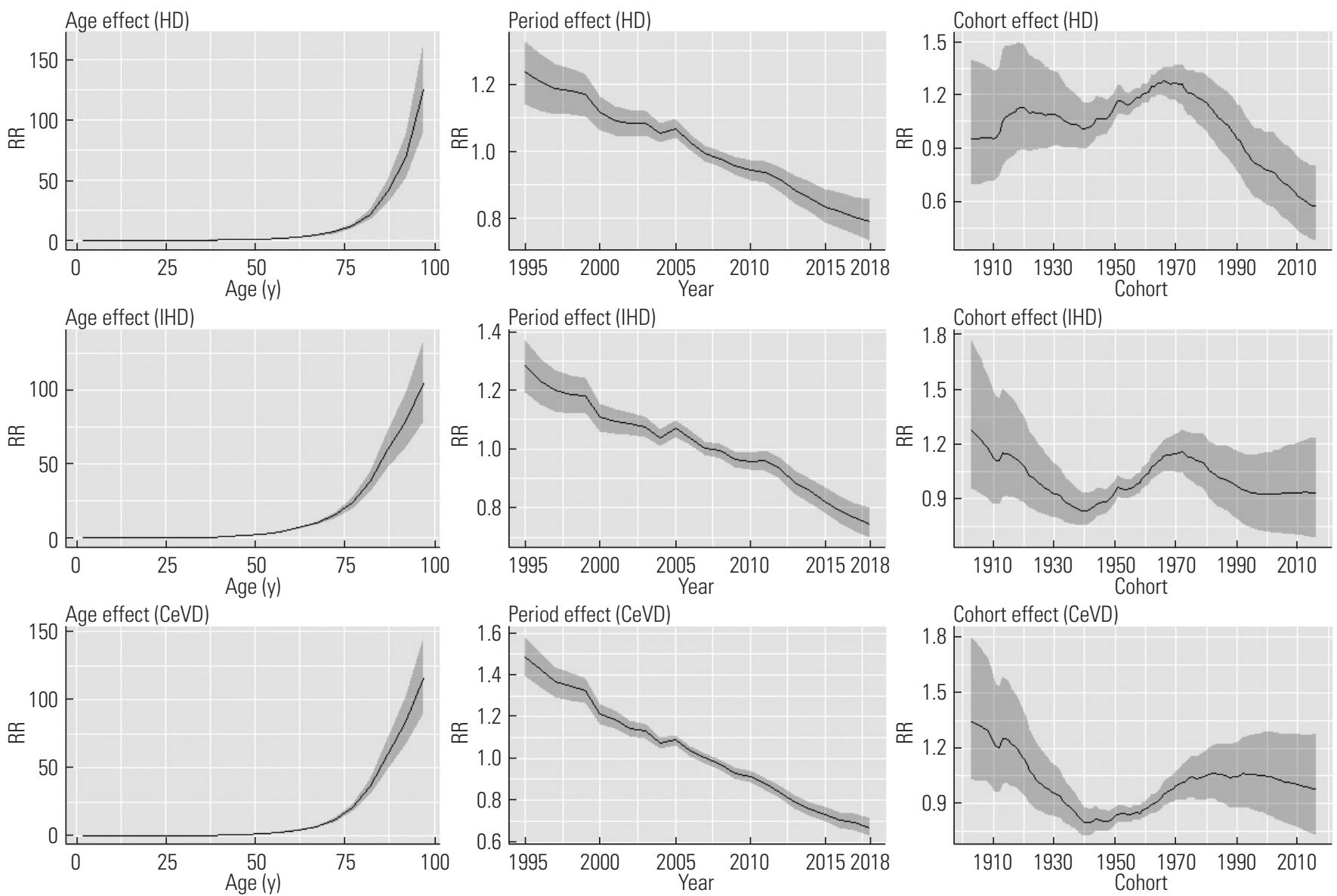

Figure 2. Results of age-period-cohort analysis of heart disease (HD), ischemic heart disease (IHD), and cerebrovascular disease (CeVD) for men. The $y$-axis indicates the rate ratio (RR) among the effects. Solid lines signify estimates of each effect, and the shadings show credible intervals of each effect. A 95\% credible interval indicates the interval where the true value of the estimate is included with a probability of $95 \%$.

age effects was more extensive than that of the other effects for all the diseases. The mortality rates of these diseases increase substantially with age, and age has the most significant effect on mortality. Although the shapes of the age effects between men and women were similar, the age effect became stronger for women than it did for men as age increased. This reflects the increasing mortality rate in women with age (at around 75 years or older). Although the overall mortality rate for women was lower than men, as shown in Figure 1, the difference in mortality among age groups was larger for women than men. In other words, the mortality rates in younger women were particularly low.

In a previous study that investigated mortality due to all CVDs through an APC analysis, using data from 1950 to 2010 [13], the period effect on CVDs as a whole was shown to decrease. In the present study, a decreased period effect was ob- served for each type of CVD. The gross mortality rate of HD increased (Supplemental Material 1), and it is considered that the incidence of non-IHD HD increased. These trends are thought to have resulted from the increased proportion of older people in the population, as the age-adjusted mortality rate of HD decreased (Figure 1). As the results of the APC analyses show, the scale of age effects was more significant for HD than for the other diseases. Therefore, the gross mortality rate of HD is affected more by aging than those of IHD and CeVD.

In contrast, the cohort effects were different when analyzed by disease and gender. In particular, the cohort effects of HD were different between men and women, which is considered to be partially related to the IHD results. Although the cohort effect on IHD increased substantially for men from cohorts born around 1940, the magnitude of the increase was smaller for women, and this affected the HD results. Furthermore, the 

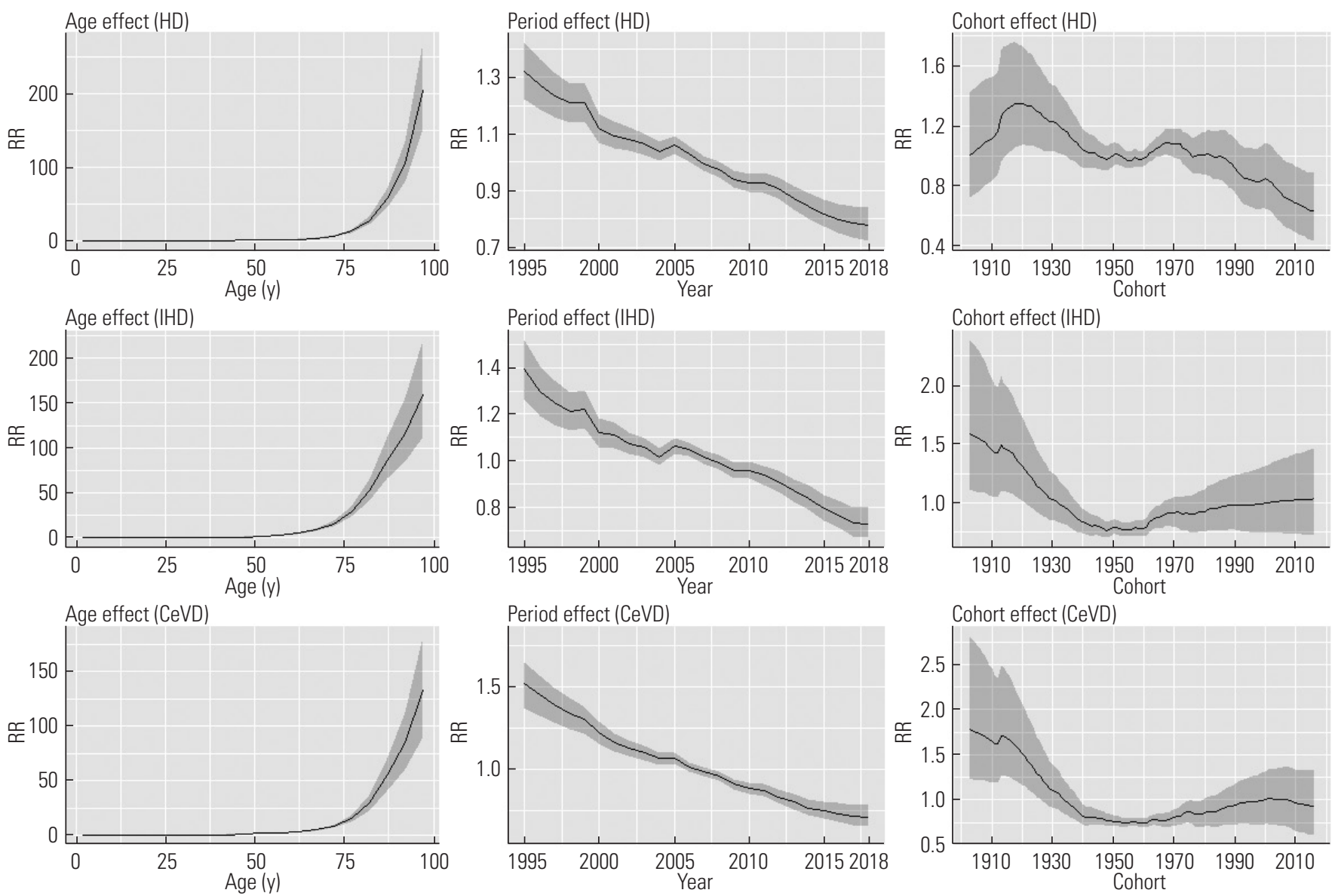

Figure 3. Results of age-period-cohort analysis of heart disease (HD), ischemic heart disease (IHD), and cerebrovascular disease $(C e V D)$ for women. The $y$-axis indicates the rate ratio (RR) among the effects. Solid lines signify estimates of each effect, and the shadings show credible intervals of each effect. A 95\% credible interval indicates the interval where the true value of the estimate is included with a probability of $95 \%$.

cohort effects on HD for both men and women began to decrease starting with cohorts born around 1970, but the magnitude of the decrease was more substantial for men, and this was also related to the effects of IHD. An APC analysis for HD has not been conducted previously in Japan, and our findings indicate that the cohort effects decreased in the cohorts born in the 1970s or later for both men and women.

According to a previous study that investigated mortality from 1955 to 2000 for IHD [2], the cohort effect decreased in the cohorts born from 1928 to 1968 for men and from 1923 to 1968 for women. Our results deviate slightly from that study's results, as after the decrease in the cohort effects for those born in the early 20th century, we observed increasing trends starting in 1940 for men and starting in 1960 for women. Several reasons may account for the difference from the previous study. First, the statistical models differed between these stud- ies. As aforementioned, the trend of estimated age effects in the previous study was not consistent with the data, which indicate a noticeable increase in the mortality rate with age. Therefore, there is a possibility that the statistical model used in the previous study did not model the data appropriately. In addition, the time period analyzed in this study is different from the period analyzed in the previous study, and it is possible that cohort effects changed as time advanced. The cohort effects experienced by particular cohorts can change in response to changes in the lifestyles of the cohort, and differences in the analyzed periods could lead to different trends being observed. In a previous study that investigated mortality due to CeVD from 1960 to 2000 [10], a greater cohort effect was also observed in cohorts born after 1948 for men and in cohorts born after 1958 for women. This result is relatively consistent with those of our study. However, in this study, we 
found that the increase stopped in the cohorts born in around 1980 for men, but it continued in subsequent cohorts for women. The turning points of the cohort effects were relatively consistent between IHD and CeVD for men and women in this study.

Compared with the decreased cohort effects of HD, those of IHD and CeVD were weaker for men; the decrease was not evident for women. The reason for this could be that CeVD and IHD are diseases that are strongly affected by individual lifestyle factors, and they have different characteristics from HD in general. The mortality rate in the younger population is higher for HD than it is for IHD or CeVD, and the apparent decline in the cohort effects of HD reflects a decrease of the mortality rate in younger ages. It is often challenging to implement preventive methods to tackle mortality as a result of HD at a younger age, which includes congenital diseases. However, the increased use of electrocardiography in routine care may help identify younger patients who have congenital diseases, and therefore could prevent sudden deaths. Additionally, more public awareness of the risk of heart failure in patients who have underlying diseases, such as hypertension or obesity, is an effective strategy for prevention [18]. According to a previous study that investigated the prevalence of obesity from 1995 to 2010 [19], the cohort effect showed a decreasing trend for the cohorts born from 1962 to 1971 for men, but showed an increasing trend for the cohorts born from 1965 to 1974 for women, which may align with our study's findings. Therefore, further APC analyses should be conducted regarding the exercise and dietary habits of both men and women. Furthermore, the difference between the genders is partially related to the substantial increase in the cohort effects for men in cohorts born between 1940 and 1970. However, this study suggests that the cohort effects for women did not decrease, as a gradually increasing trend was found in cohorts born around the 1960s or later, and a preventive approach against IHD and CeVD, particularly for women, is therefore needed.

A limitation of this study is that the overall accuracy of causes of death listed in Vital Statistics has not been reported in Japan. According to a study that investigated the accuracy of death certificates in a hospital in Japan, there is not a high level of accurate reporting for heart disease being a cause of death [20]. The accuracy of cause of death reporting is considered to depend on the disease. This highlights the need for a future validation study to be conducted to better understand the accuracy of cause of death reporting. Another limitation is that we used data from the younger population for the analysis. The cohort effects identified in this population at the time of the analysis may change in the future, making it vitally important to conduct APC analyses regularly to understand trends in cohort effects.

\section{SUPPLEMENTAL MATERIALS}

Supplemental materials are available at https://doi.org/10. 3961/jpmph.20.037.

\section{CONFLICT OF INTEREST}

The author has no conflicts of interest associated with the material presented in this paper.

\section{FUNDING}

None.

\section{ACKNOWLEDGEMENTS}

Enago has proofread the manuscript.

\section{AUTHOR CONTRIBUTIONS}

All work was done by TO.

\section{ORCID}

Tasuku Okui https://orcid.org/0000-0001-5098-8502

\section{REFERENCES}

1. Ministry of Health, Labour and Welfare of Japan. Overview of annual total monthly report of the vital statistics 2018 [cited 2020 Feb 10]. Available from: https://www.mhlw.go.jp/toukei/ saikin/hw/jinkou/geppo/nengai18/index.html (Japanese).

2. Ma E, Iso H, Takahashi H, Yamagishi K, Tanigawa T. Age-periodcohort analysis of mortality due to ischemic heart disease in Japan, 1955 to 2000. Circ J 2008;72(6):966-972.

3. Lee HA, Park H. Trends in ischemic heart disease mortality in Korea, 1985-2009: an age-period-cohort analysis. J Prev Med Public Health 2012;45(5):323-328. 
4. Ocaña-Riola R, Mayoral-Cortés JM, Fernández-Ajuria A, Sánchez-Cantalejo C, Martín-Olmedo P, Blanco-Reina E. Age, period, and cohort effects on mortality from ischemic heart disease in southern Spain. Rev Esp Cardiol (Engl Ed) 2015;68(5): 373-381.

5. Gero K, Eshak ES, Ma E, Takahashi H, Noda H, Iso H. Health disparities in ischaemic heart disease mortality in Hungary from 1970 to 2010: an age-period-cohort analysis. J Epidemiol 2015; 25(6):399-406.

6. Ministry of Health, Labour and Welfare of Japan. Annual report on health, labor and welfare 2018 [cited 2020 Feb 10]. Available from: https://www.mhlw.go.jp/stf/wp/hakusyo/kousei/18/index.html (Japanese).

7. Ogata S, Nishimura K, Guzman-Castillo M, Sumita Y, Nakai M, Nakao YM, et al. Explaining the decline in coronary heart disease mortality rates in Japan: contributions of changes in risk factors and evidence-based treatments between 1980 and 2012. Int J Cardiol 2019;291:183-188.

8. Hisamatsu T, Miura K. Trends of mortality rate and incidence rate of cardiovascular disease in Japan. Jpn J Cardiovasc Dis Prev 2018;53(1):1-8 (Japanese).

9. Smith TR, Wakefield J. A review and comparison of age-period-cohort models for cancer incidence. Stat Sci 2016;31(4): 591-610.

10. Ma E, Takahashi H, Mizuno A, Okada M, Yamagishi K, Iso H. Stratified age-period-cohort analysis of stroke mortality in Japan, 1960 to 2000. J Stroke Cerebrovasc Dis 2007;16(3):91-102.

11. Ocaña-Riola R, Blanco-Reina E, Navarro-Moreno E, MayoralCortés JM. Age-period-cohort effects on mortality from cerebrovascular disease in southern Spain. J Stroke Cerebrovasc Dis 2014;23(9):2274-2282.

12. Wang Z, Hu S, Sang S, Luo L, Yu C. Age-period-cohort analysis of stroke mortality in China: data from the global burden of disease study 2013. Stroke 2017;48(2):271-275.

13. Maruo S, Iso H. Trends in cohort effects of cardiovascular disease mortality in Japan, 1950-2010. Nihon Koshu Eisei Zasshi 2015;62(2):57-65 (Japanese).

14. Ministry of Health, Labour and Welfare of Japan. The vital statistics: e-Stat General counter of government statistics [cited 2020 Feb 10]. Available from: https://www.e-stat.go.jp/statsearch $/$ files?page $=1 \&$ layout $=$ datalist \&toukei $=00450011 \&$ ts tat $=000001028897 \&$ cycle $=7 \&$ tclass $1=000001053058 \&$ tclas s2 $=000001053061 \&$ tclass3 $=000001053072$ (Japanese).

15. Ministry of Health, Labour and Welfare of Japan. Overview of the vital statistics survey [cited 2020 Feb 27]. Available from: https://www.mhlw.go.jp/toukei/list/81-1b.html (Japanese).

16. Saito I, Aono H, Ikebe T, Ozawa H, Yamashita T. Change in the mortality statistics for heart disease in Japan: an influence of the 10th revision of the International Classification of Diseases. Jpn J Cardiovasc Dis Prev 1998;33(4):257-265 (Japanese).

17. Naing NN. Easy way to learn standardization: direct and indirect methods. Malays J Med Sci 2000;7(1):10-15.

18. Tsutsui $H$, Isobe $M$, Ito $H$, Ito $H$, Okumura $K$, Ono M, et al. Guidelines for diagnosis and treatment of acute and chronic heart failure (JCS 2017/JHFS 2017) [cited 2020 Feb 10]. Available from: http://www.j-circ.or.jp/guideline/pdf/JCS2017_tsutsui_ h.pdf (Japanese).

19. Yamakita M, Uchida H, Kawamura K, Homma T, Odagiri Y. Effects of age, period, and cohort on the trends in obesity rate and energy intake ratio from fat in Japanese adults. Nihon Koshu Eisei Zasshi 2014;61(8):371-384. (Japanese).

20. Mieno MN, Tanaka N, Arai T, Kawahara T, Kuchiba A, Ishikawa S, et al. Accuracy of death certificates and assessment of factors for misclassification of underlying cause of death. J Epidemiol 2016;26(4):191-198. 